\title{
Iron deficiency during pregnancy is associated with a reduced risk of adverse birth outcomes in a malaria-endemic area in a longitudinal cohort study
}

Freya J. I. Fowkes ${ }^{1,2,34^{*}}$, Kerryn A. Moore ${ }^{1,3}$, D. Herbert Opi ${ }^{1,5}$, Julie A. Simpson ${ }^{3}$, Freya Langham 1,2, Danielle I. Stanisic ${ }^{6}$, Alice Ura ${ }^{7}$, Christopher L. King ${ }^{8}$, Peter M. Siba ${ }^{7}$, Ivo Mueller ${ }^{9,10}$, Stephen J. Rogerson ${ }^{11}$ and James G. Beeson ${ }^{1,4,11^{*}}$

\begin{abstract}
Background: Low birth weight (LBW) and preterm birth (PTB) are major contributors to infant mortality and chronic childhood morbidity. Understanding factors that contribute to or protect against these adverse birth outcomes is an important global health priority. Anaemia and iron deficiency are common in malaria-endemic regions, but there are concerns regarding the value of iron supplementation among pregnant women in malaria-endemic areas due to reports that iron supplementation may increase the risk of malaria. There is a lack of evidence on the impact of iron deficiency on pregnancy outcomes in malaria-endemic regions.

Methods: We determined iron deficiency in a cohort of 279 pregnant women in a malaria-endemic area of Papua New Guinea. Associations with birth weight, LBW and PTB were estimated using linear and logistic regression. A causal model using sequential mediation analyses was constructed to assess the association between iron deficiency and LBW, either independently or mediated through malaria and/or anaemia.

Results: Iron deficiency in pregnant women was common (71\% at enrolment) and associated with higher mean birth weights ( $230 \mathrm{~g}$; $95 \%$ confidence interval, Cl 118, 514; $p<0.001$ ), and reduced odds of LBW (adjusted odds ratio, $\mathrm{aOR}=0.32 ; 95 \% \mathrm{Cl} 0.16,0.64 ; p=0.001)$ and PTB $(\mathrm{aOR}=0.57 ; 95 \% \mathrm{Cl} 0.30,1.09 ; p=0.089)$. Magnitudes of effect were greatest in primigravidae (birth weight $351 \mathrm{~g} ; 95 \% \mathrm{Cl}$ 188, 514; $p<0.001$; LBW aOR 0.26; 95\% Cl 0.10, 0.66; $p=0.005$; PTB $\mathrm{aOR}=0.39,95 \% \mathrm{Cl} 0.16,0.97 ; p=0.042$ ). Sequential mediation analyses indicated that the protective association of iron deficiency on LBW was mainly mediated through mechanisms independent of malaria or anaemia.

Conclusions: Iron deficiency was associated with substantially reduced odds of LBW predominantly through malaria-independent protective mechanisms, which has substantial implications for understanding risks for poor pregnancy outcomes and evaluating the benefit of iron supplementation in pregnancy. This study is the first longitudinal study to demonstrate a temporal relationship between antenatal iron deficiency and improved birth outcomes. These findings suggest that iron supplementation needs to be integrated with other strategies to prevent or treat infections and undernutrition in pregnancy to achieve substantial improvements in birth outcomes.
\end{abstract}

Keywords: Iron deficiency, Low birth weight, Preterm birth, Malaria, Anaemia, Pregnancy, Plasmodium falciparum

\footnotetext{
* Correspondence: freya.fowkes@burnet.edu.au; beeson@burnet.edu.au

${ }^{1}$ Burnet Institute, Maternal and Child Health Program, Life Sciences and

Public Health, Melbourne, VIC 3004, Australia

Full list of author information is available at the end of the article
}

(c) The Author(s). 2018 Open Access This article is distributed under the terms of the Creative Commons Attribution 4.0 International License (http://creativecommons.org/licenses/by/4.0/), which permits unrestricted use, distribution, and reproduction in any medium, provided you give appropriate credit to the original author(s) and the source, provide a link to the Creative Commons license, and indicate if changes were made. The Creative Commons Public Domain Dedication waiver (http://creativecommons.org/publicdomain/zero/1.0/) applies to the data made available in this article, unless otherwise stated. 


\section{Background}

Low birth weight (LBW; birth weight $<2500 \mathrm{~g}$ ) and preterm birth (PTB; <37 weeks gestation) are major contributors to infant mortality and chronic childhood morbidity. LBW is associated with $>80 \%$ of infant deaths [1]. Understanding factors that moderate or exacerbate the burden of LBW and PTB is, therefore, an important global health priority. Poor nutrition, anaemia and infectious diseases are common among pregnant women globally, particularly in resource-limited settings, and are important contributors to LBW. Iron deficiency (defined by the World Health Organisation [WHO] as ferritin $<15 \mu \mathrm{g} / \mathrm{L}$ [2]) is a common feature of undernutrition and a major cause of anaemia and it contributes to an estimated global burden of 600,000 perinatal and 100,000 maternal deaths per year [3]. Many regions with a high burden of undernutrition and anaemia are also endemic for malaria and approximately 125 million women living in malaria-endemic areas become pregnant each year $[4,5]$. Malaria contributes to anaemia by causing haemolysis and dyserythropoiesis and it is also a major cause of LBW and PTB [6, 7].

Despite the high prevalence of undernutrition and anaemia among pregnant women in resource-limited and malaria-endemic settings, there is limited knowledge on the contribution of iron deficiency and other specific preventable deficiencies to LBW and poor pregnancy outcomes. To reduce the burden of anaemia and poor pregnancy outcomes, the WHO recommends that all pregnant women receive daily iron and folate supplements (30-60 mg of elemental iron plus $400 \mu \mathrm{g}$ of folic acid), in addition to "measures to prevent, diagnose and treat malaria" in malaria-endemic areas [8]. However, there are concerns regarding the value of antenatal iron supplementation in malaria-endemic areas due to reports of harmful interactions between iron and malaria [9]. Moreover, the link between iron deficiency and poor pregnancy outcomes in malaria-endemic and tropical regions, and the value of iron supplementation on improving birth outcomes, is unclear.

Iron deficiency has been associated with reduced highdensity Plasmodium falciparum parasitaemia, associated clinical symptoms of malaria and reduced placental $P$. falciparum infection in case-control and cross-sectional studies of pregnant women living in malaria-endemic areas [10]. Iron supplements given to children in malaria-endemic areas have been associated with increased parasitaemia, risk of clinical malaria and risk of severe illness and death (not specific to malaria) in some controlled trials [11, 12]. However, clinical trials have shown inconsistent associations between daily iron supplementation and risk of peripheral or placental $P$. falciparum infection in Africa [10,13-15]. One study reported an increased risk of $P$. vivax after supplementation in Asia [10]. There is also no clear evidence for the association between iron supplementation and birth outcomes in pregnant women living in areas where they are at risk of malaria [10, 13-16]. A recent trial in west African pregnant and non-pregnant women found an increased rate of adverse events among those receiving iron supplementation [17]. These issues are compounded by a lack of understanding of how iron deficiency, anaemia and malaria may interact to influence birth outcomes. This knowledge is essential to the evidence base of iron supplementation programmes in malaria-endemic and resource-limited settings where there is a high burden of infectious diseases and poor nutrition.

The objectives of this study were to determine the association between iron deficiency and birth outcomes, and to quantify how malaria mediated these associations, in a longitudinal study of pregnant women in a malariaendemic area of Papua New Guinea (PNG), which has the largest population at risk of anaemia, undernutrition malaria and poor birth outcomes in the South-West Pacific region.

\section{Methods \\ Study design}

Pregnant women $(n=470)$ were enrolled into a longitudinal study of malaria in pregnancy in Madang Province, PNG, conducted through the PNG Institute of Medical Research (Additional file 1: Methods) [18]. Briefly, study participation occurred in parallel with clinic attendance at the first antenatal visit, at 30-34 weeks' gestation and at delivery. Pregnant women were enrolled at their first antenatal consultation at Alexishafen Health Centre between September 2005 and October 2007. At enrolment and delivery, $5 \mathrm{ml}$ of peripheral blood was collected, and stored at $-20{ }^{\circ} \mathrm{C}$ or $-70{ }^{\circ} \mathrm{C}$. Gestational age at enrolment was estimated from fundal height $(\mathrm{cm})$ and haemoglobin was measured using a HemoCue haemoglobinometer (Hemocue, Ängelholm, Sweden). Women with haemoglobin concentrations $<5 \mathrm{~g} / \mathrm{dL}$ were referred for appropriate care and were not enrolled in the study. All women were prescribed ferrous sulfate $270 \mathrm{mg}$ and folic acid $0.3 \mathrm{mg}$ daily, according to local policy; women with $\mathrm{Hb} 5-7 \mathrm{~g} / \mathrm{dL}$ were prescribed two tablets daily. Intermittent preventative treatment in pregnancy with sulfadoxinepyrimethamine (IPTp-SP; $1500 \mathrm{mg} / 75 \mathrm{mg}$ respectively) was recommended to all women according to national guidelines. At delivery, intervillous placental blood and placental tissue samples were collected. Birth weight was measured using SECA baby scales and gestational age was estimated from Ballard scores performed by the study nurse, following training by a paediatrician [18]. Ethical approval was granted by the PNG Medical Research Advisory Council and the Alfred Health Human Research Ethics Committee, and written informed consent was obtained from all study participants. Of the 470 women 
enrolled into the initial study [18], 376 women completed follow-up to delivery and 97 women were excluded from the current analysis ( 5 multiple births; 43 newborns were not seen within 3 days after delivery; 1 data entry error; 31 with insufficient blood sample; and 17 women with missing birth outcome data) (Additional file 1). Therefore, 279 women were included in this analysis (Table 1). Included and excluded women did not differ in the distribution of enrolment variables (Additional file 1: Table S1).

\section{Exposures and outcomes}

Exposures of interest were iron stores (ferritin) and iron deficiency (ferritin $<15 \mu \mathrm{g} / \mathrm{L}$ ) at enrolment. Birth outcomes of interest were birth weight (grams), LBW $(<2500 \mathrm{~g})$ and PTB (<37 weeks gestation). Malaria outcomes of interest were peripheral $P$. falciparum or P. vivax spp. infection [by polymerase chain reaction (PCR) or light microscopy] and placental $P$. falciparum infection (detected by light microscopy or placental histology).

\section{Laboratory procedures}

Peripheral and placental Plasmodium spp. infection was determined by light microscopy of Giemsa-stained thick and thin blood films, and PCR, as previously described [18]. Among women who completed follow-up to delivery, ferritin concentrations were measured in serum samples (collected at enrolment) by established ELISA with reference controls (Immunology Consultants Laboratory Immunoperoxidase Assay). C-reactive protein (CRP) levels were determined using established ELISA kits with reference controls (Elisakit). The ferritin and CRP intra-assay and inter-assay coefficients of variation were all $<10 \%$.

\section{Statistical analysis}

Changes in Plasmodium spp. prevalence and haemoglobin within an individual woman between enrolment and delivery were quantified using McNemar's chi-square test or Student's paired $t$-test, respectively. Associations between ferritin (transformed to log base 2) and iron deficiency and birth outcomes (birth weight, LBW and PTB) were estimated using multivariable linear and logistic regression, respectively. Confounding variables identified a priori [gravidity, maternal education, maternal mid upper arm circumference (MUAC) at enrolment (as a marker of undernutrition), maternal smoking and gestational age] were adjusted for in multivariable regression models. Red blood cell genetic polymorphisms (SAO, CR1 and $\alpha$-thalassaemia) were not included in the models, as we have previously shown in this cohort that they are not associated with risk of Plasmodium infection or birth weight [18]. Newborn sex, a predictor of birth outcomes, was also included in the multivariable linear regression models to potentially reduce the residual standard deviation, and thus increase the precision
Table 1 Participant characteristics, malaria parameters, iron markers and delivery outcomes

\begin{tabular}{|c|c|}
\hline Variable & $\begin{array}{l}\text { Mean }[S D] \text {, range; or median } \\
\{\mid \mathrm{QR}\} \text {, range; or } N(\%)\end{array}$ \\
\hline \multicolumn{2}{|l|}{$\overline{\text { Participant characteristics at enrolment }^{1}}$} \\
\hline Maternal age, years $(N=268)$ & $24[17], 16-49$ \\
\hline $\begin{array}{l}\text { Mid upper arm circumference, } \mathrm{cm} \\
(N=270)\end{array}$ & $22.4[1.8], 12-30$ \\
\hline Primigravidae & 106/279 (38) \\
\hline $\begin{array}{l}\text { Estimated gestational age, weeks } \\
(N=276)\end{array}$ & $25.3[4.2], 7-36$ \\
\hline \multicolumn{2}{|l|}{ Education } \\
\hline None or primary & $154 / 275(56)$ \\
\hline Secondary+ & $121 / 275(44)$ \\
\hline Current smoker (yes) & $56 / 278(20)$ \\
\hline Used bed net last night (yes) & 200/264 (76) \\
\hline $\begin{array}{l}\text { Clinical history of fever, chills or } \\
\text { headache in past } 7 \text { days (yes) }\end{array}$ & $67 / 276(24)$ \\
\hline Palpable spleen (yes) & $51 / 262(19)$ \\
\hline \multicolumn{2}{|l|}{ Malariometrics and iron status at enrolment } \\
\hline $\begin{array}{l}\text { Plasmodium spp. infection detectable } \\
\text { by PCR }\end{array}$ & 185/279 (66) \\
\hline Plasmodium spp. infection ${ }^{2}$ & $98 / 279(35)$ \\
\hline P. falciparum & $93 / 279(33)$ \\
\hline P. vivax & 9/279 (3) \\
\hline Haemoglobin, g/dL ( $N=279)$ & $8.5[1.4], 5.3-12.8$ \\
\hline Ferritin, $\mu \mathrm{g} / \mathrm{L}(N=279)$ & $8.2\{4.6-17.5\}, 2.4-121.3$ \\
\hline Iron deficient (ferritin < 15 mg/L) & 199/279 (71) \\
\hline $\mathrm{CRP}, \mathrm{mg} / \mathrm{L}(\mathrm{N}=258)^{3}$ & $\begin{array}{l}5.15\{1.80-11.72\} \\
0.18-255.65\end{array}$ \\
\hline High CRP (> 10 mg/L) & $90 / 279(32)$ \\
\hline \multicolumn{2}{|c|}{ Malariometric and birth outcomes at delivery } \\
\hline Placental Plasmodium spp. infection ${ }^{2}$ & $39 / 223(17)$ \\
\hline P. falciparum & $34 / 223(15)$ \\
\hline P. vivax & $6 / 223(3)$ \\
\hline Plasmodium spp. infection ${ }^{2}$ & $37 / 274(13)$ \\
\hline P. falciparum & $31 / 274(11)$ \\
\hline P. vivax & $6 / 274(2)$ \\
\hline Haemoglobin, g/dL $(N=270)$ & $9.2[1.7], 4.7-14.2$ \\
\hline Birth weight, grams $(N=279)$ & $2857[452], 1400-4500$ \\
\hline Low birth weight $(<2500 \mathrm{~g})$ & $47 / 279(17)$ \\
\hline $\begin{array}{l}\text { Gestational age at delivery, weeks } \\
(N=279)\end{array}$ & $38\{37-40\}, 28-42$ \\
\hline Preterm birth (<37 weeks gestation) & $62 / 279(22)$ \\
\hline
\end{tabular}

${ }^{1} N=279$

${ }^{2}$ Plasmodium spp. infection detectable by light microscopy of Giemsa-stained thick and thin blood films, unless otherwise specified. Four women had a mixed infection at enrolment ( $P$. falciparum and $P$. vivax), one woman had mixed placental infection and one woman had mixed peripheral infection at delivery

${ }^{3} 21$ measurements were outside the assay standard curve so were excluded from the CRP concentration descriptive analysis but classified as $>10 \mathrm{mg} / \mathrm{L}$ for high CRP descriptive analysis

$C R P C$-reactive protein, $I Q R$ interquartile range, $P C R$ polymerase chain reaction, $S D$ standard deviation 
of our parameter estimates for iron exposures. Effect modification by gravidity (primigravid/multigravid) was investigated by including an interaction term between iron exposures and gravidity; $p$ values for interactions were derived from likelihood ratio tests comparing models including and excluding interaction terms. We performed sequential mediation analyses to estimate marginal natural direct and indirect effects for the association between iron deficiency and LBW mediated through: (1) peripheral infection detected by light microscopy or PCR, (2) placental $P$. falciparum infection detected by light microscopy or histology, and (3) anaemia, by calculating potential outcomes from logistic models with inverse probability weighting to achieve balance in the iron deficiency groups in terms of the confounders (gravidity, maternal education, maternal mid-upper arm circumference at enrolment, gestational age). We obtained 95\% confidence intervals (CIs) for marginal natural direct effects and marginal natural indirect effects by bootstrapping using the percentile method and 1000 replications. All statistical analyses were performed using Stata Version 13 (StataCorp, College Station, TX, USA).

\section{Results}

\section{Iron deficiency and malaria in the study population}

At enrolment (first antenatal visit), the median maternal age was 24 years (interquartile range, IQR 21-28), the mean estimated gestational age was 25 weeks (standard deviation, SD 4.2) and $62 \%$ of women were multigravidae. Reported use of a bed net (to prevent malaria) during the night before enrolment was $76 \%$. For clinical symptoms, $24 \%$ of women had a history of fever, headache or chills within the 7 days prior to enrolment, and 19\% had a palpable spleen (Table 1). Plasmodium spp. infection at enrolment was common; $35 \%$ and $66 \%$ of women had peripheral Plasmodium spp. parasitaemia detectable by light microscopy and PCR, respectively. The majority (95\%) of infections were $P$. falciparum.

At enrolment, haemoglobin levels were generally very low (mean $8.5 \mathrm{~g} / \mathrm{dL}, \mathrm{SD} 1.4$ ) and the prevalence of anaemia was very high. Overall, 95\% (266/279) of women had anaemia $(\mathrm{Hb}<11 \mathrm{~g} / \mathrm{dL}), 61 \%(171 / 279)$ had moderate anaemia $(\mathrm{Hb}<9 \mathrm{~g} / \mathrm{dL})$ and $12 \%(33 / 279)$ had severe anaemia $(\mathrm{Hb}<7 \mathrm{~g} / \mathrm{dL})$. Furthermore, ferritin concentrations were low (median 8.2 $\mu \mathrm{g} / \mathrm{L}$; IQR 4.6-17.5) and iron deficiency (defined as ferritin $<15 \mu \mathrm{g} / \mathrm{L}$ according to the WHO) was present in $71 \%$ of women at enrolment (Table 1). The prevalence was $62 \%$ and $77 \%$ in primigravid and multigravid women respectively. Elevated CRP can be a marker of an acute phase response that may also raise ferritin levels and potentially lead to misclassification of iron-deficient women as iron-replete. In our study, elevated CRP (>10 mg/L) was found in 46 (57\%) iron-replete women and 44 (22\%) iron-deficient women $(p<0.001)$. When potentially misclassified women were excluded (women with raised CRP and ferritin $>15$ ), the prevalence of iron deficiency was $85 \%$. Ferritin and CRP levels were only weakly correlated (Spearman's rho, $\rho=0.245 ; 95 \%$ CI $0.127,0.357 ; p<0.001)$. There was no correlation between haemoglobin at enrolment and ferritin at enrolment $(\rho=0.035 ; 95 \%$ CI $-0.083,0.152 ; p=0.562)$, and no association between iron deficiency and anaemia at enrolment, suggesting that multiple factors contributed to anaemia, aside from iron deficiency. We found that 96\% (77/80) of iron-replete and 95\% (189/199) of iron-deficient women were anaemic $(p=0.648), 69 \%(55 / 80)$ of iron-replete and 58\% (116/199) of iron-deficient women had moderate anaemia $(p=0.105)$, and $9 \%(7 / 80)$ of iron-replete and $13 \%(26 / 199)$ of iron-deficient women had severe anaemia $(p=0.313)$.

At delivery, 13\% of women had peripheral parasitaemia detectable by light microscopy (a significant decrease from enrolment, $p<0.001$ ), and mean haemoglobin was $9.2 \mathrm{~g} / \mathrm{dL}$ (SD 1.7), a significant increase from enrolment, with a mean difference of -0.66 (95\% CI $-0.86,-0.45 ; p<0.001)$ (Table 1). Placental parasitaemia was present in $17 \%$ by light microscopy (18\% and $17 \%$ in primigravidae and multigravidae, respectively) (Table 1).

\section{Associations between iron deficiency and birth outcomes} Mean birth weight in the cohort was $2857 \mathrm{~g}$ (SD $452 \mathrm{~g}$ ) and median (IQR) gestational age was 38 weeks (37-40 weeks). Poor pregnancy outcomes were common, with $17 \%$ being LBW and $22 \%$ of babies born preterm $(<37$ weeks estimated gestation) (Table 1). Surprisingly, lower ferritin levels and iron deficiency were associated with higher mean birth weights in multivariable regression analyses (Table 2). For every twofold increase in ferritin, the estimated adjusted mean decrease in birth weight was $-63 \mathrm{~g}$ (95\% CI -103, -123; $p=0.002$ ), and iron-deficient women gave birth to newborns that were $230 \mathrm{~g}$ heavier than newborns of iron-replete women (95\% CI 118, 342; $p<0.001$; Table 2). There was evidence of effect modification by gravidity, whereby the reduction in mean birth weight associated with higher iron stores was greatest in primigravidae (Table 2). Among primigravidae, birth weight was $351 \mathrm{~g}$ higher among iron-deficient (95\% CI 188, 514), compared to iron-replete women $(p<0.001)$, whereas among multigravidae, birth weight was $125 \mathrm{~g}$ higher among iron-deficient women $(95 \%$ CI $-28,277 ; p=0.108$; Table 2).

Iron deficiency was associated with a $68 \%$ reduction in the odds of LBW (adjusted odds ratio, aOR $=0.32$; $95 \%$ CI $0.16,0.64 ; p=0.001$ ), and a $43 \%$ reduction in the odds of preterm birth, although the association with PTB had weak statistical significance $(\mathrm{aOR}=0.57 ; 95 \%$ CI 0.30 , 
Table 2 Associations between iron deficiency and birth outcomes and effect modification by gravidity

\begin{tabular}{|c|c|c|c|}
\hline \multicolumn{4}{|l|}{ Birth weight (grams) } \\
\hline Iron stores & Unadjusted mean difference $(95 \% \mathrm{Cl}) ; p$ & Adjusted mean difference $(95 \% \mathrm{Cl}) ; p$ & \\
\hline \multirow[t]{3}{*}{ Ferritin, $\mu \mathrm{g} / \mathrm{L}\left(\log _{2}\right)^{1}$} & $-72(-113,-30) ; 0.001$ & $-63(-103,-23) ; 0.002$ & \\
\hline & & Primigravid & Multigravid \\
\hline & & $-114(-173,-55) ;<0.001$ & $-20(-74,33) ; 0.457$ \\
\hline \multicolumn{4}{|l|}{ Iron deficiency } \\
\hline Iron replete & Reference group & Reference group & \\
\hline \multirow[t]{3}{*}{ Iron deficient } & $204(89,320) ; 0.001$ & $230(118,342) ;<0.001$ & \\
\hline & & Primigravid & Multigravid \\
\hline & & $351(188,514)_{;}<0.001$ & $125(-28,277) ; 0.108$ \\
\hline \multicolumn{4}{|c|}{ Low birth weight $(<2500 \mathrm{~g})$} \\
\hline Iron stores & Unadjusted OR (95\% Cl); $p$ & Adjusted OR (95\% Cl); $p$ & \\
\hline \multicolumn{4}{|l|}{ Iron deficiency } \\
\hline Iron replete & Reference group & Reference group & \\
\hline \multirow[t]{3}{*}{ Iron deficient } & $0.34(0.18,0.65) ; 0.001$ & $0.32(0.16,0.64) ; 0.001$ & \\
\hline & & Primigravid & Multigravid \\
\hline & & $0.26(0.10,0.66) ; 0.005$ & $0.42(0.15,1.20) ; 0.105$ \\
\hline \multicolumn{4}{|c|}{ Preterm birth (<37 weeks) } \\
\hline Iron stores & Unadjusted OR (95\% Cl); $p$ & Adjusted OR (95\% Cl); $p$ & \\
\hline \multirow[t]{3}{*}{ Ferritin, $\mu \mathrm{g} / \mathrm{L}\left(\log _{2}\right)^{1}$} & $1.13(0.91,1.41) ; 0.254$ & $1.06(0.84,1.35) ; 0.605$ & \\
\hline & & Primigravid & Multigravid \\
\hline & & $1.20(0.86,1.66) ; 0.280$ & $0.93(0.65,1.33) ; 0.681$ \\
\hline \multicolumn{4}{|l|}{ Iron deficiency } \\
\hline Iron replete & Reference group & Reference group & \\
\hline \multirow[t]{3}{*}{ Iron deficient } & $0.60(0.33,1.10) ; 0.098$ & $0.57(0.30,1.09) ; 0.089$ & \\
\hline & & Primigravid & Multigravid \\
\hline & & $0.39(0.16,0.97) ; 0.042$ & $0.86(0.32,2.30) ; 0.767$ \\
\hline
\end{tabular}

Multivariable models including confounding variables (gravidity, gestational age, education, mid-upper arm circumference and smoking), and sex of the newborn (linear models only). ${ }^{1}$ Ferritin transformed to log base 2 due to positively skewed distribution; coefficients are, therefore, for the absolute or relative change in outcome associated with each twofold increase in ferritin concentration. $p$ values for gravidity interaction parameters are 0.019 (ferritin, birth weight), 0.042 (iron deficiency, birth weight), 0.500 (iron deficiency, LBW), 0.299 (ferritin, PTB) and 0.236 (iron deficiency, PTB)

$\mathrm{Cl}$ confidence interval, $L B W$ low birth weight, OR odds ratio, PTB preterm birth

1.09; $p=0.089$ ). An analysis of effect modification also showed that the magnitudes of effect between iron deficiency and LBW were highly significant and greater in primigravidae (LBW aOR 0.26; 95\% CI 0.10, 0.66; $p=$ 0.005 ) than in multigravidae (LBW aOR $0.42 ; 95 \% \mathrm{CI}$ $0.15,1.20 ; p=0.105$; Table 2 ). An analysis of effect modification also showed that the association between iron deficiency and PTB was restricted to primigravidae (aOR 0.39; 95\% CI 0.16, 0.97; $p=0.042$ ) compared to multigravidae (aOR 0.86; 95\% CI 0.32, 2.30; $p=0.767$; Table 2). Additionally, we performed analyses excluding iron-replete women who had evidence of inflammation (ferritin $>15 \mu \mathrm{g} / \mathrm{ml}$ and CRP $>10 \mathrm{mg} / \mathrm{L}$ ), that is women who may have been misclassified as iron replete due to elevated ferritin resulting from an acute phase response. This did not alter our results and a similar association between iron deficiency and birth weight was found (Additional file 1).
Investigating iron deficiency, malaria and anaemia as causes of LBW using mediation analyses

To investigate the causes of the association between iron deficiency and LBW further, we explored the association between iron deficiency and anaemia $(\mathrm{Hb}<9 \mathrm{~g} / \mathrm{dL})$, peripheral malaria infection and placental malaria and whether these variables mediated the association between iron deficiency and LBW. Iron deficiency was associated with a $48 \%$ reduction in moderate anaemia at enrolment (aOR 0.52; 95\% CI 0.27, 0.97; $p=0.040$, adjusted for gravidity, education, smoking, MUAC and gestational age), reflective of multiple contributors to anaemia in the study population. Iron deficiency was also associated with a $56 \%$ reduction in peripheral blood parasitaemia at enrolment as detected by light microscopy $(\mathrm{aOR}=0.44 ; 95 \%$ CI $0.25,0.79 ; p=0.006)$ and a $67 \%$ reduction in PCR-detected infection (aOR 0.33; 95\% 
CI $0.17,0.67 ; p=0.002$ ), but no association was found with placental malaria $(\mathrm{aOR}=0.85 ; 95 \%$ CI $0.38,1.90$; $p=0.695)$. However, these associations were confounded by women potentially misclassified as iron replete. After excluding these potentially misclassified women, there were no significant protective associations between iron deficiency and anaemia $(\mathrm{aOR}=0.77$; 95\% CI $0.33,1.79 ; p=0.543$ ), peripheral parasitaemia (light microscopy aOR 1.47 ; 95\% CI 0.59, 3.68; $p=0.406$; PCR aOR 0.66 ; $95 \%$ CI $0.28,1.56 ; p=0.343)$ or placental malaria $(\mathrm{aOR}=1.60 ; 95 \%$ CI 0.43, 5.87; $p=0.480)$.

We used sequential mediation analyses to assess the effects of iron deficiency on LBW, either directly or mediated through placental $P$. falciparum malaria, anaemia or peripheral malaria at enrolment. Sequential mediation analyses were performed to quantify the proportion of the observed protective effect of iron deficiency on LBW that was mediated through malaria and anaemia, accounting for potential confounders (maternal education, MUAC, gravidity, smoking and gestational age) (Table 3 ). The mediators were malaria at enrolment by light microscopy, moderate to severe anaemia at enrolment $(\mathrm{Hb}$ $<9 \mathrm{~g} / \mathrm{dL}$ ), placental malaria at delivery by light microscopy (directed acyclic graph shown in Fig. 1). The mediation analyses are sequential because there were multiple mediators that are not independent of each other (Fig. 1). In sequential mediation analyses, mediation through placental malaria only can be determined as it immediately precedes the outcome (LBW). Malaria detected at enrolment precedes other mediators of interest (anaemia and placental malaria) and therefore, mediation solely through malaria detected at enrolment cannot be determined, only what is mediated through malaria at enrolment and anaemia and placental malaria.

Interestingly, there was evidence for a substantial direct protective effect of iron deficiency on risk of LBW that was not mediated through protection against placental malaria, anaemia or malaria present in peripheral blood (risk ratio $=0.44 ; 95 \%$ CI $0.25,0.79$ ). In contrast, only $7 \%$ of the association between iron deficiency and LBW was mediated through placental $P$. falciparum malaria and only $12 \%$ was mediated through pathways that included peripheral malaria infection, which included indirect pathways via anaemia and placental malaria (Table 3). Similar effects were seen in sensitivity analyses whereby women with raised CRP levels who may have been misclassified as iron replete (ferritin $>15 \mu \mathrm{g} / \mathrm{ml}$ and CRP $>10 \mathrm{mg} / \mathrm{L}$ ) were excluded from analyses (Table 3). Furthermore, similar associations were found in analyses investigating the mediation through malaria and anaemia detected at the second antenatal visit (30-34 weeks gestation) and/or placental malaria defined by placental histology (Additional file 1). In summary, these analyses suggest that the protective association of iron deficiency on LBW is largely mediated through mechanisms independent of malaria and anaemia.

\section{Discussion}

LBW and PTB are major risk factors for infant morbidity and mortality and identifying factors that contribute to these risks, or protect against them, is crucial for advancing child health globally. Furthermore, concerns regarding potentially harmful malaria-iron interactions have raised questions about the appropriateness of antenatal iron supplementation for anaemia in malaria-endemic populations [9]. Here, we provide the first evidence of a protective association of maternal iron deficiency on LBW, and to a lesser extent PTB, in a malaria-endemic area where iron deficiency and anaemia are common, and elucidated the potential effect of malaria-iron interactions on birth outcomes. Iron deficiency was associated with substantially reduced risks of LBW, and this beneficial effect appeared largely mediated through mechanisms that are independent of an interaction between iron deficiency and malaria. The apparent protective effect of iron deficiency was greatest in primigravid women, a high-risk

Table 3 Mediation of association between iron deficiency and low birth weight

\begin{tabular}{|c|c|c|c|}
\hline Mediating variables & Natural direct effect, risk ratio $(95 \% \mathrm{Cl})$ & Natural indirect effect, risk ratio $(95 \% \mathrm{Cl})$ & Proportion indirect \\
\hline \multicolumn{4}{|l|}{ All women } \\
\hline Placental malaria only & $0.47(0.25,0.83)$ & $0.92(0.63,1.25)$ & $7 \%$ \\
\hline Anaemia and placental malaria & $0.45(0.25,0.79)$ & $0.88(0.60,1.18)$ & $10 \%$ \\
\hline Malaria, anaemia and placental malaria & $0.44(0.25,0.79)$ & $0.87(0.69,1.39)$ & $12 \%$ \\
\hline \multicolumn{4}{|l|}{ Sensitivity analyses* } \\
\hline Placental malaria only & $0.34(0.17,0.79)$ & $1.09(0.67,1.80)$ & $4 \%$ \\
\hline Anaemia and placental malaria & $0.33(0.17,0.78)$ & $1.07(0.63,1.86)$ & $4 \%$ \\
\hline Malaria, anaemia and placental malaria & $0.33(0.17,0.78)$ & $1.06(0.65,1.84)$ & $3 \%$ \\
\hline
\end{tabular}

Numbers are risk ratios (95\% confidence interval). Natural direct effect is the effect of iron deficiency on birth outcome, not mediated through the specified mediators. Natural indirect effect is the effect of iron deficiency on birth outcome, mediated through the specified mediator. $\mathrm{Cl}$ confidence interval

*Women potentially misclassified as iron deficient (ferritin $>15$ and C-reactive protein $>10)$ are excluded from analysis $(N=46)$ 


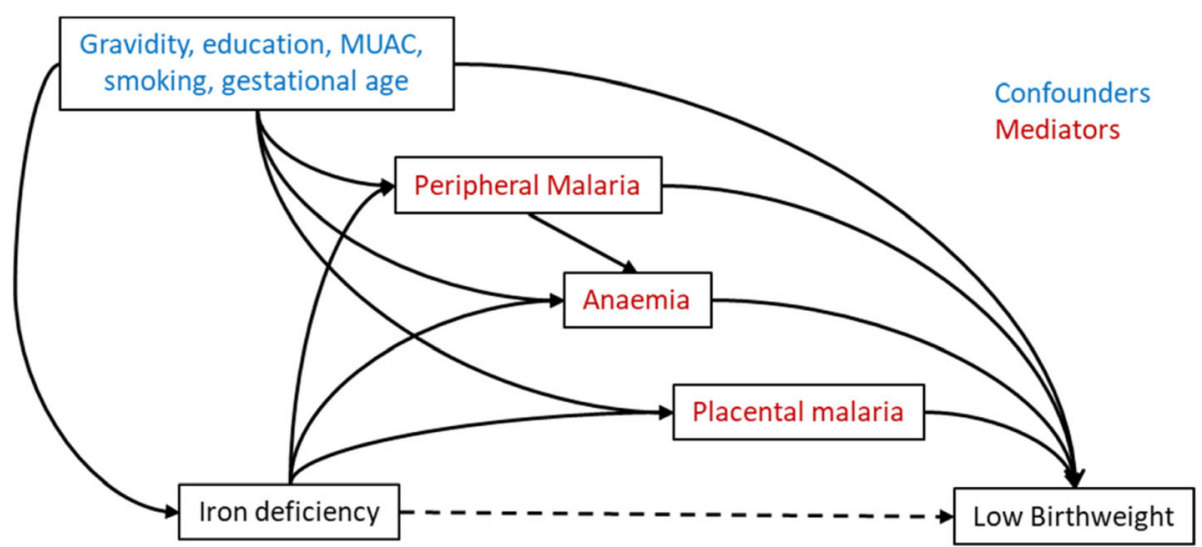

Fig. 1 Directed acyclic graph for the mediated association between iron deficiency and low birth weight. Sequential mediation analyses were performed to assess the effects of iron deficiency on low birth weight, directly or mediated through malaria at enrolment by light microscopy, moderate anaemia at enrolment $(\mathrm{Hb}<9 \mathrm{~g} / \mathrm{dL})$ and placental malaria at delivery by light microscopy. Gravidity, maternal education, mid-upper arm circumference, gestational age and smoking were considered confounders. The results of the analyses are shown in Table 3. MUAC mid upper arm circumference

group who are more susceptible to, and more severely affected by, a range of infectious diseases [19].

We demonstrated that the associations between iron deficiency and malaria infection were confounded by the presence of an acute phase response (raised CRP). Iron deficiency was associated with reduced malaria infection when the analysis included all women. However, when women who were potentially misclassified as iron replete (ferritin $>15$ but raised CRP levels) were excluded, no protective association was found. Women with malaria are more likely to have raised CRP and therefore, more likely to be misclassified as iron replete. As a result, the analyses would more likely demonstrate an apparent protective effect of iron deficiency unless these effects are considered in analyses. Previously published analyses of cross-sectional and case-control studies of associations between iron deficiency and peripheral and placental P. falciparum infection of women [10] have included these potentially misclassified women, which may have biased results towards finding an association between iron deficiency and reduced malaria risk, or increased the magnitude of the protective effect. There is some biological plausibility to protective associations of iron deficiency, as in vitro studies have shown that iron deficiency can limit the development of blood-stage infection by starving the parasite of exoerythrocytic forms of iron [20-23], impair merozoite invasion and propagation [24], or increase the immune-mediated clearance of infected erythrocytes [25, 26]. However, a direct causal mechanism between iron deficiency and placental malaria independent of the aforementioned reductions in peripheral infection is yet to be proposed and we found no association between iron deficiency and placental malaria in this study.

A strength of our study is that we used sequential mediation analysis as a valuable approach to estimate the contribution of potential causal relationships. This is the first analysis to quantify the mediation of the effect of iron deficiency on birth outcomes through malaria (both peripheral and placental) and anaemia. This is also the first mediation analysis of the effect of iron deficiency on birth outcomes to account for both exposure-outcome and mediator-outcome confounders, which is a source of bias in traditional mediation analysis [27]. Importantly, we found that only $12 \%$ of the protective effect of iron deficiency on LBW was mediated through malaria or anaemia. Iron deficiency was not significantly associated with anaemia or peripheral or placental malaria, which are key determinants of LBW and PTB in this population [18]. This may explain the lack of mediation through placental malaria in this cohort study and further studies using sequential mediation analyses are warranted in other populations. After accounting for mediation through peripheral Plasmodium spp. infection and placental $P$. falciparum infection, we found that iron deficiency was still associated directly with a $>50 \%$ reduction in the odds of LBW. Host iron is essential to a range of other bacteria, parasites and viruses, and iron deficiency may also inhibit the development of these pathogens [28-30]. Therefore, reductions in infectious pathogens due to iron deficiency may explain the association between iron deficiency and improved birth outcomes in our study. In PNG, there is a high burden of disease from bacterial and viral pathogens, including respiratory, gastrointestinal and sexually transmitted infections [31], that may be influenced by iron status [30]. In PNG, a higher prevalence of these infections is also found in primigravid compared to multigravid women [32], which may also explain why larger magnitudes of the effect of iron deficiency on birth outcomes were observed in high risk primigravid women. Other infections 
may also be important. A recent study of iron supplementation in non-pregnant African women found higher rates of treatment for gastrointestinal infections in women receiving iron [17]. Further studies are needed to understand the mechanisms by which iron deficiency leads to improved birth weight, and this knowledge would help identify appropriate strategies to reduce LBW while also reducing the burden of iron deficiency and anaemia. Examining the effects of iron deficiency on other infectious pathogens common in pregnancy, together with their interactions with malaria and anaemia, is a priority for future studies.

This study is the first longitudinal study globally to demonstrate a temporal relationship between antenatal iron deficiency and birth outcomes, and together with the sequential mediation analyses, provides significant causal evidence for the associations observed. In PNG, malaria transmission is high and both $P$. falciparum and $P$. vivax are endemic. The majority of infections $(>95 \%)$ in our study were $P$. falciparum and therefore, our findings may be more broadly applicable to other high $P$. falciparum transmission areas, such as sub-Saharan Africa. However further studies are required to determine the consistency of our findings and the effect size across areas of varying prevalence of iron deficiency, malaria and other infections and in areas where the relative contribution of the multiple factors that cause iron deficiency differs. Research that identifies populations or settings where iron deficiency associations with birth weight are strongest would be valuable in guiding public health policy. In our study, we experienced a 40\% loss to follow-up; however, given that the characteristics of the women lost to follow-up did not differ from those of the included women (Additional file 1), the loss is unlikely to have affected the internal validity of the study. While the specific causes of iron deficiency have not been defined, these are likely to include low dietary intake as well as infections such as hookworm and malaria that can impact iron absorption or utilisation.

Iron deficiency was defined according to the WHO standard definition (ferritin $<15 \mu \mathrm{g} / \mathrm{L}$ for non-pregnant women) [2]. Ferritin is also an acute phase protein that may increase during inflammation independently of iron stores [33]. Previous cross-sectional and case-control studies of iron deficiency and malaria in pregnancy either did not measure inflammation or reported that a proportion of women had inflammation and their reported associations may have been confounded [34, 35]. In our study, we performed sensitivity analyses to show that the protective associations observed between iron deficiency and birth weight were not confounded by the presence of inflammation. In the absence of bone marrow biopsies, ferritin remains the gold standard to assess iron deficiency [36], and although other markers of iron deficiency have been explored, such as the soluble transferrin receptor, they currently lack established definitions for iron deficiency in pregnancy. Any potential bias associated with misclassification of true iron status with the use of ferritin for defining iron deficiency is unlikely to change the conclusions of this study given the magnitude and statistical strength of the observed associations and the biological plausibility.

\section{Conclusions}

Our findings show that iron deficiency in pregnancy is associated with a reduced risk of LBW and PTB that is not simply explained by a potential protective effect against malaria. The mechanism(s) underlying this paradox in malaria-endemic and resource-limited settings with high burdens of infectious diseases and poor nutrition are not yet well understood but are essential to elucidate given the importance and scale of this global public health issue and that LBW and PTB are important determinants of poor infant outcomes. Our results highlight that it is essential to provide both supplementation for anaemia and effective malaria prophylaxis during pregnancy, and future implementation trials to improve the uptake of these interventions as well as trials of integrated interventions for nutrition, malaria and other infections linked with LBW and PTB are warranted. Integration of screening and prophylaxis for other infectious diseases and poor nutrition, alongside current interventions in antenatal care, may be needed to achieve substantial improvements in birth and infant outcomes.

\section{Additional file}

Additional file 1: Supplementary methods and analyses. (DOCX 142 kb)

Abbreviations

aOR: Adjusted odds ratio; Cl: Confidence intervals; CRP: C-reactive protein; Hb: Haemoglobin; IQR: Inter-quartile range; LBW: Low birthweight;

MUAC: Mid upper arm circumference; OR: Odds Ratio; PCR: Polymerase chain reaction; PNG: Papua New Guinea; PTB: Pre-term birth; SD: Standard deviation; WHO: World Health Organization

\section{Acknowledgements}

We thank all the study participants, Francesca Baiwog and the staff of the Alexishafen Health Centre under the direction of Sister Valsi Kurian for their enthusiastic cooperation with the study, and the staff of the PNG Institute of Medical Research for assistance with microscopy, data entry and study administration.

\section{Funding}

Funding was provided by the Australian Research Council (future fellowship to FJIF), the National Health and Medical Research Council, Australia (program grant and senior research fellowship to JGB and JAS). The Burnet Institute is supported by the Independent Research Institutes Infrastructure Support Scheme of the National Health and Medical Research Council and a Victoria State Government Operational Infrastructure Support grant. Funding bodies had no role in the design of the study, the collection, analysis and interpretation of the data or in writing the manuscript. 


\section{Availability of data and materials}

The data analysed during the current study are not publicly available due to study participants' privacy, but additional de-identified data are available from the corresponding authors on reasonable request.

\section{Authors' contributions}

FJIF, FL, DHO and JGB performed and interpreted the iron-deficiency assays. CLK, PMS, IM, SJR and JGB conceived and designed the cohort study. DIS and AU performed the fieldwork. FJIF, KAM and JAS analysed the data and drafted the report. All authors approved the final report.

\section{Ethics approval and consent to participate}

Ethical approval was granted by the PNG Medical Research Advisory Council and the Alfred Health Human Research Ethics Committee, and written informed consent was obtained from all study participants.

\section{Consent for publication}

As part of the ethics approval, the participants provided consent to allow the results of this research work to be published.

\section{Competing interests}

The authors declare that they have no competing interests.

\section{Publisher's Note}

Springer Nature remains neutral with regard to jurisdictional claims in published maps and institutional affiliations.

\section{Author details}

${ }^{1}$ Burnet Institute, Maternal and Child Health Program, Life Sciences and Public Health, Melbourne, VIC 3004, Australia. ²Department of Epidemiology and Preventive Medicine, Monash University, Melbourne, VIC 3008, Australia. ${ }^{3}$ Centre for Epidemiology and Biostatistics, Melbourne School of Population and Global Health, The University of Melbourne, Melbourne, VIC 3010, Australia. ${ }^{4}$ Department of Infectious Diseases, Central Clinical School, Monash University, Melbourne, VIC 3004, Australia. ${ }^{5}$ Department of Immunology, Monash University, Central Clinical School, Melbourne, VIC 3004, Australia. ${ }^{6}$ Institute for Glycomics, Griffith University, South Brisbane, QLD 4101, Australia. ${ }^{7}$ Papua New Guinea Institute of Medical Research, Goroka, EHP, Papua New Guinea. ${ }^{8}$ Center for Global Health and Diseases, Case Western Reserve University, Cleveland, OH, USA. ${ }^{2}$ Walter and Eliza Hall Institute of Medical Research, Parkville, VIC 3050, Australia. ${ }^{10}$ Department of Parasites and Insect Vectors, Institut Pasteur, 75015 Paris, France. ${ }^{11}$ Department of Medicine (RMH), The University of Melbourne, Parkville, VIC 3010, Australia.

\section{Received: 14 February 2018 Accepted: 3 August 2018}

\section{Published online: 20 September 2018}

\section{References}

1. Lawn JE, Cousens S, Zupan J. 4 million neonatal deaths: when? Where? Why? Lancet. 2005;365(9462):891-900.

2. WHO. Serum ferritin concentrations for the assessment of iron status and iron deficiency in populations, vol. 2011. Geneva; 2011. p. 1-5.

3. Are We Making Progress on Reducing Anemia in Women? Cross-country Comparison of Anemia Prevalence, Reach, and Use of Antenatal Care and Anemia Reduction Interventions. A2Z: The USAID Micro-nutrient and Child Blindness Project. http://www.a2zproject.org/pdf/ReducingAnemia_low_res_ 06212011.pdf.

4. Desai M, Kuile FO, Nosten F, Mcgready R, Asamoa K, Brabin B, Newman RD. Epidemiology and burden of malaria in pregnancy. Lancet Infect Dis. 2007;7:93-104.

5. Dellicour S, Tatem AJ, Guerra CA, Snow RW, ter Kuile FO. Quantifying the number of pregnancies at risk of malaria in 2007: a demographic study. PLOS Med. 2010;7(1):e1000221.

6. Guyatt HL, Snow RW. Impact of malaria during pregnancy on low birth weight in sub-Saharan Africa. Clin Microbiol Rev. 2004;17(4):760-9. table of contents

7. Walker PG, ter Kuile FO, Garske T, Menendez C, Ghani AC. Estimated risk of placental infection and low birthweight attributable to Plasmodium falciparum malaria in Africa in 2010: a modelling study. Lancet Glob Health. 2014;2(8):e460-7.

8. WHO. Guideline: Daily iron and folic acid supplementation in pregnant women, vol. 2012. Geneva; 2012.
9. Schumann K, Solomons NW. Can iron supplementation be reconciled with benefits and risks in areas hyperendemic for malaria? Food Nutr Bull. 2013; 34(3):349-56.

10. Sangaré L, van Eijk AM, Ter Kuile FO, Walson J, Stergachis A. The association between malaria and Iron status or supplementation in pregnancy: a systematic review and meta-analysis. PLoS One. 2014;9:e87743.

11. Oppenheimer S, Gibson F, Macfarlane S, et al. Iron supplementation increases prevalence and effects of malaria: report on clinical studies in Papua New Guinea. Trans R Soc Trop Med Hyg. 1986;80:603-12.

12. Sazawal S, Black RE, Ramsan M, Chwaya HM, Stoltzfus RJ, Dutta A, Dhingra U, Kabole I, Deb S, Othman MK, et al. Effects of routine prophylactic supplementation with iron and folic acid on admission to hospital and mortality in preschool children in a high malaria transmission setting: community-based, randomised, placebo-controlled trial. Lancet. 2006; 367:133-43

13. Mwangi MN, Roth JM, Smit MR, Trijsburg L, Mwangi AM, Demir AY, Wielders $J$ J, Mens PF, Verweij JJ, Cox SE, et al. Effect of daily antenatal Iron supplementation on Plasmodium infection in Kenyan women: a randomized clinical trial. JAMA. 2015;314(10):1009-20.

14. Etheredge AJ, Premji Z, Gunaratna NS, Abioye Al, Aboud S, Duggan C, Mongi R, Meloney L, Spiegelman D, Roberts D, et al. Iron supplementation in Iron-replete and nonanemic pregnant women in Tanzania: a randomized clinical trial. JAMA Pediatr. 2015;169(10):947-55.

15. Pena-Rosas JP, De-Regil LM, Garcia-Casal MN, Dowswell T. Daily oral iron supplementation during pregnancy. Cochrane Database Syst Rev. 2015;7: CD004736.

16. Pena-Rosas JP, De-Regil LM, Gomez Malave H, Flores-Urrutia MC, Dowswell T. Intermittent oral iron supplementation during pregnancy. Cochrane Database Syst Rev. 2015;10:Cd009997.

17. Brabin L, Roberts SA, Gies S, Nelson A, Diallo S, Stewart CJ, Kazienga A, Birtles J, Ouedraogo S, Claeys Y, et al. Effects of long-term weekly iron and folic acid supplementation on lower genital tract infection - a double blind, randomised controlled trial in Burkina Faso. BMC Med. 2017;15(1):206.

18. Stanisic DI, Moore KA, Baiwog F, Ura A, Clapham C, King CL, Siba PM, Beeson JG, Mueller I, Fowkes FJ, et al. Risk factors for malaria and adverse birth outcomes in a prospective cohort of pregnant women resident in a high malaria transmission area of Papua New Guinea. Trans R Soc Trop Med Hyg. 2015;109(5):313-24.

19. Jamieson DJ, Theiler RN, Rasmussen SA. Emerging infections and pregnancy. Emerg Infect Dis. 2006;12(11):1638-43.

20. Raventos-Suarez C, Pollack S, Nagel RL. Plasmodium falciparum: inhibition of in vitro growth by desferrioxamine. Am J Trop Med Hyg. 1982:31(5):919-22.

21. Ferrer $P$, Tripathi AK, Clark MA, Hand CC, Rienhoff HY Jr, Sullivan DJ Jr. Antimalarial iron chelator, FBS0701, shows asexual and gametocyte Plasmodium falciparum activity and single oral dose cure in a murine malaria model. PLoS One. 2012;7(5):e37171.

22. Pollack S. Effects of iron and desferrioxamine on the growth of Plasmodium falciparum in vitro. Br J Haematol. 1987;65(2):256-7.

23. Pollack S, Rossan RN, Davidson DE, Escajadillo A. Desferrioxamine suppresses Plasmodium falciparum in Aotus monkeys. Proc Soc Exp Biol Med. 1987; 184(2):162-4.

24. Clark MA, Goheen MM, Fulford A, Prentice AM, Elnagheeb MA, Patel J, Fisher N, Taylor SM, Kasthuri RS, Cerami C. Host iron status and iron supplementation mediate susceptibility to erythrocytic stage Plasmodium falciparum. Nat Commun. 2014;5:4446.

25. Matsuzaki-Moriya C, Tu L, Ishida H, Imai T, Suzue K, Hirai M, Tetsutani K, Hamano S, Shimokawa C, Hisaeda H. A critical role for phagocytosis in resistance to malaria in iron-deficient mice. Eur J Immunol. 201 1;41(5):1365-75.

26. Weiss G, Werner-Felmayer G, Werner ER, Grunewald K, Wachter H, Hentze MW. Iron regulates nitric oxide synthase activity by controlling nuclear transcription. J Exp Med. 1994;180(3):969-76.

27. Richiardi L, Bellocco R, Zugna D. Mediation analysis in epidemiology: methods, interpretation and bias. Int J Epidemiol. 2013;42(5):1511-9.

28. Skaar EP. The battle for iron between bacterial pathogens and their vertebrate hosts. PLoS Pathog. 2010;6(8):e1000949.

29. Noinaj N, Buchanan SK, Cornelissen CN. The transferrin-iron import system from pathogenic Neisseria species. Mol Microbiol. 2012;86(2): 246-57.

30. Cross JH, Bradbury RS, Fulford AJ, Jallow AT, Wegmuller R, Prentice AM, Cerami C. Oral iron acutely elevates bacterial growth in human serum. Sci Rep. 2015;5:16670. 
31. Vallely A, Page A, Dias S, Siba P, Lupiwa T, Law G, Millan J, Wilson DP, Murray JM, Toole M, et al. The prevalence of sexually transmitted infections in Papua New Guinea: a systematic review and meta-analysis. PLoS One. 2010;5(12):e15586

32. Vallely LM, Toliman P, Ryan C, Rai G, Wapling J, Tomado C, Huliafi S, Munnull G, Rarau P, Phuanukoonnon S, et al. Prevalence and risk factors of chlamydia trachomatis, Neisseria gonorrhoeae, trichomonas vaginalis and other sexually transmissible infections among women attending antenatal clinics in three provinces in Papua New Guinea: a cross-sectional survey. Sex Health. 2016;13(5):420-7.

33. Gabay C, Kushner I. Acute-phase proteins and other systemic responses to inflammation. N Engl J Med. 1999;340(6):448-54.

34. Senga EL, Harper G, Koshy G, Kazembe PN, Brabin BJ. Reduced risk for placental malaria in iron deficient women. Malar J. 2011;10:47.

35. Kabyemela ER, Fried M, Kurtis JD, Mutabingwa TK, Duffy PE. Decreased susceptibility to Plasmodium falciparum infection in pregnant women with Iron deficiency. J Infect Dis. 2008;198:163-6.

36. Wheeler S. Assessment and interpretation of micronutrient status during pregnancy. Proc Nutr Soc. 2008;67(4):437-50.

Ready to submit your research? Choose BMC and benefit from:

- fast, convenient online submission

- thorough peer review by experienced researchers in your field

- rapid publication on acceptance

- support for research data, including large and complex data types

- gold Open Access which fosters wider collaboration and increased citations

- maximum visibility for your research: over $100 \mathrm{M}$ website views per year

At BMC, research is always in progress.

Learn more biomedcentral.com/submissions 